\title{
The role of contextual cues in the haptic perception of orientations and the oblique effect
}

\author{
MARION LUYAT and CHRISTINE MORONI \\ Université Charles-de-Gaulle-Lille 3, Villeneuve d'Ascq, France \\ and \\ EDOUARD GENTAZ \\ UMR 5105 CNRS, Université Pierre Mendès France, Grenoble, France
}

\begin{abstract}
Blindfolded right-handed participants were asked to position, with the right hand, a frontoparallel rod to one of three orientations: vertical $\left(0^{\circ}\right)$ and left $45^{\circ}$ and right $45^{\circ}$ obliques. Simultaneously, three different backgrounds were explored with the left hand: smooth, congruent stripes (parallel to the orientation to be produced), or incongruent stripes (tilted relative to the orientation to be produced). The analysis of variable errors showed that the oblique effect (higher precision for the vertical orientation than for the oblique orientations) was weakened in the presence of contextual cues, because of an improvement in oblique precision. Moreover, the analysis of constant errors revealed that the perception of orientations erred in the direction of the stripes, similar to the effect that has been found with vision, where visual contextual cues (tilted frame or lines) divert the perception of the vertical. These results are discussed in relation to a patterncentric frame of reference hypothesis or as a congruency effect.
\end{abstract}

In this study, we address the question of whether contextual cues are integrated in the haptic (tactualkinesthetic) perception of orientations by humans. Previous experiments have provided clear evidence that orientation processing differs according to the value of the orientation. Indeed, the vertical orientation is perceived more precisely than oblique orientations (for reviews, see Gentaz, 2000; Gentaz \& Ballaz, 2000; Gentaz \& Tschopp, 2002). This anisotropy, called the oblique effect by Appelle (1972), is present whatever the perceptual system involved in the perceptual judgment. It has been found with a great variety of tasks in visual (Attneave \& Olson, 1967; Furmanski \& Engel, 2000; Gentaz et al., 2001; Luyat \& Gentaz, 2002; McIntyre, Lipshits, Zaoui, Berthoz, \& Gurfinkel, 2001; McMahon \& MacLeod, 2003; Westheimer, 2003), haptic (Appelle \& Countryman, 1986; Gentaz, Badan, Luyat, \& Touil, 2002; Gentaz \& Hatwell, 1995, 1996, 1998, 1999; Gentaz \& Streri, 2004; Hermens \& Gielen, 2003; Kappers, 1999; Kappers \& Koenderink, 1999; Lechelt \& Verenka, 1980; Luyat, Gentaz, Regia Corte, \& Guerraz, 2001), and somatovestibular (Gentaz et al., 2001) systems.

The oblique effect in haptics has usually been shown with a reproduction-of-orientations task in which blind-

This work was supported by grants from the "Cognitique, ACI" program, Ministère de la Recherche (cog. nos. 95 and 78B), and the MSH program provided by the University of Lille 3. We thank Aurelie Delperdange, Fabien Brunet, Eoghan Ó Duill, and the Laboratory of Psychology and Neurocognition of Grenoble for their assistance, as well the participants for their help in this research. Correspondence should be addressed to M. Luyat, Laboratoire URECA, UFR de Psychologie, Université Lille 3, Pont de Bois, BP 149, 59653 Villeneuve d'Ascq, France (e-mail: marion.luyat@univ-lille3.fr). folded participants had to scan a rod with one hand and to reproduce the previous orientation with the same or the contralateral hand (Appelle \& Countryman, 1986; Gentaz \& Hatwell, 1995, 1996, 1998, 1999; Lechelt \& Verenka, 1980). Evidence for a similar oblique effect has also been shown with a production task (Gentaz et al., 2002) in which a rod had to be oriented to a given orientation (vertical, horizontal, or oblique) following verbal instructions and without previous coding of the tested orientation. In contrast to the reproduction of orientations that have been previously explored but not verbally defined, performance on the production task appears more likely to be based on internal models of spatial orientations, as has been suggested by previous researchers (Heeley \& Buchanan-Smith, 1990; McIntyre et al., 2001; Morgan, 1991).

Essock (1980) has proposed a distinction between "Class 1 oblique effects," observed when one is measuring the basic functioning of the visual system (acuity or contrast threshold), and "Class 2 oblique effects," observed in paradigms in which cognitive processes involved in identifying, remembering, or categorizing the orientations of stimuli are emphasized. Class 1 effects can be explained consistently by the properties of orientationselective neurons (Li, Peterson, \& Freeman, 2003; Westheimer, 2003) and may be tied to a retinocentric frame of reference (Banks \& Stolarz, 1975; Chen \& Levi, 1996; Corwin, Moskowitz-Cook, \& Green, 1977; Furmanski \& Engel, 2000; Lennie, 1974; Orban, Vandenbussche, \& Vogels, 1984; Saarinen \& Levi, 1995). The origin of Class 2 oblique effects is less clear. Essock, Krebs, and Prather (1997) suggested that Class 2 oblique effects can be explained by a general bias in the representation of 
oblique stimuli, which may stem from the fact that any oblique orientation can be encoded in relation to the vertical and horizontal norms that define a reference frame, whereas the vertical and horizontal orientations can be encoded directly (Foster \& Westland, 1998; Gentaz, 2000; Regan \& Price, 1986). In this perspective, identifying the nature of the spatial reference frame in which the orientations are mapped should make a better understanding of the origin of the Class 2 visual oblique effect possible.

Different reference frames can be selected to define an orientation in space. Classically, a broad distinction has been made between spatial frames that are egocentric (with the participant's body as reference) and those that are allocentric (with environmental cues as references; for reviews, see Howard, 1982; Rock, 1990; Wade, 1992). Several egocentric reference frames can be defined: (1) a retinocentric reference frame specifically relevant for visual tasks, (2) head- or trunk-centered reference frames implied in both visual and haptic tasks, and (3) a hand/shoulder-centered reference frame that can be relevant for haptic tasks. The allocentric reference frame can be divided into a gravitational frame, defined by the direction of the gravity pull (geocentric frame), and patterncentric reference frames, defined by visual or haptic contextual cues. In natural conditions, the gravitational, body-centered, and visual reference frames are most often aligned. Consequently, the visual and haptic oblique effects could result from either egocentric or allocentric mapping of orientations. However, in the absence of visual or tactual contextual cues, tilting the head or the body uncouples the gravitational and the egocentric reference frames, which then allows one to specify the reference frame with respect to which orientations are defined (for a review, see Luyat et al., 2001).

Adopting this perspective and using a reproductionof-orientation task in haptics (Luyat et al., 2001) and in vision (Luyat \& Gentaz, 2002), we have found that the oblique effect was mapped in neither a gravitational nor an egocentric reference frame but, rather, in a subjective gravitational frame of reference that was tilted in the same direction as that in which the head or body was tilted. Actually, the oblique effect observed in the upright posture disappeared in tilted conditions, mainly because of a decrease in the precision of the vertical and horizontal settings. In tilted conditions, the subjective vertical-the apparent gravitational vertical for each participant, which was physically oblique in the tilted postureproved to be the orientation reproduced most precisely. This frame of reference could be subject to cognitive factors such as voluntary control, as has been suggested by previous work (Attneave \& Reid, 1968).

In these experiments, the stimulus consisted of a single rod without any contextual cues around it. However, for vision, it is well known that perception of spatial orientation is enhanced when vertical contextual cues are available and, as a consequence, is strongly affected by a roll or pitch of single tilted lines or by a more struc- tured context, such as a room (Groen, Jenkin, \& Howard, 2002; Howard \& Hu, 2001; Luyat \& Ohlmann, 1997; Luyat, Ohlmann, \& Barraud, 1997). When a square frame is used to enclose a stimulus rod that is to be adjusted to the vertical, the angular function is complex, because a square frame has four major axes: the vertical and horizontal axes and, also, the two diagonal axes, which could exert an influence. Beh, Wenderoth, and Purcell (1971) have proved that for a display with a small angular size $\left(7.57^{\circ}\right)$, the judgmental bias is strongly influenced toward the axis nearest to the gravitational vertical-that is, the side or the diagonal, depending on the tilt of the frame. With a large square frame subtending an angular size of about $20^{\circ}$, the deviation of the settings seems to systematically be in the direction of the context from $0^{\circ}$ to $45^{\circ}$, with a maximum effect around $15^{\circ}$ of tilt (Spinelli, Antonucci, Goodenough, Pizzamiglio, \& Zoccolotti, 1991). Moreover, given the strong influence of visual directional cues on the perception of orientation, whether there is a possible role for the visual frame of reference in the oblique effect remains an open question.

In haptics, the effect of contextual cues on the perception of orientation has not yet been investigated, except by Walker (1972), in a short report in which the participant had to position a rod to the vertical while passing the right hand through a tilted grid. This author reported an effect, but unfortunately, both precise data and important methodological aspects, such as the amount of tilt used, were not provided. Thus, research is needed, first, to prove that contextual cues have a role in the haptic perception of orientations. This is precisely the main objective of the present research. The haptic perceptual field remains greatly reduced, as compared with the visual field, even when both hands are used with active exploratory movements, As a result, the perceptual cues that are relevant in a task (or useful as a spatial exocentric reference frame) are less available in the haptic than in the visual modality. Moreover, voluntary movements must be made in order to compensate for the smallness of the haptic perceptual field. As a result, haptic perception is highly sequential. However, the latter property permits contextual effects to be reduced in haptic perception, as compared with visual perception, in which this is not the case, because of the presence of simultaneous peripheral (contextual cues) and central (the target) stimulation. This difference is important and can explain, for example, why the Delboeuf illusion (in which a circle enclosed in another circle is perceived to be bigger than a samesized circle perceived alone) exists only in vision (see Gentaz \& Hatwell, 2004): It is not observed in haptics because the inner circle (the target) can be scanned with one hand without contact with the outer circle (contextual cues).

In the present experiment, we asked blindfolded sitting participants to use the right hand to position a rod in three different spatial orientations in the frontoparallel (vertical) plane: to the vertical and along two oblique orientations $45^{\circ}$ to the left and to the right of the vertical 
one. This production task was carried out with four different backgrounds: (1) in the absence of contextual cues (smooth), (2) in the presence of congruent contextual cues (stripes parallel to the orientation to be produced), (3) in the presence of a context of stripes tilted to the left, and (4) in the presence of a context of stripes tilted to the right of the orientation to be produced. The performances of the orientation adjustment were estimated using two independent measures: precision (variable error) and accuracy (constant error). Using the right and the left hands at the same time, with one hand exploring the context and the other adjusting the stimulus rod to the expected orientation, allows simultaneous stimulation by both the context and the stimulus. In this case, effects similar to those found with vision are more likely to be observed. More precisely, two main hypotheses were formulated. First, in the absence of contextual cues, an oblique effect could be expected, with a better precision for vertical production, relative to oblique production. Second, the contextual cues could affect the production of orientations and the oblique effect. More precisely, in the presence of congruent contextual cues, the oblique effect could be weakened, due to an enhancement of oblique production. In the presence of incongruent contextual cues, the perception of orientations could be affected in the direction of the contextual cues.

\section{METHOD}

\section{Participants}

Twenty participants ( 13 females, 7 males; mean age $=23.5$ years, $S D=3.53$, range $=19-28)$ were tested. All the participants were right-handed (as assessed with the Edinburgh scale; see Oldfield, 1971) and used their preferred hand. They gave written informed consent for their participation. All of them were completely unaware of the task and of the objective of the experiment.

\section{Materials}

The haptic apparatus was composed of a vertical grooved metallic disk (diameter, $40 \mathrm{~cm}$ ) equipped with a rod $(25 \times 1.8 \mathrm{~cm})$. This disk was engraved with stripes with a width of $3 \mathrm{~mm}$ and a depth of $0.5 \mathrm{~mm}$, with a $3-\mathrm{mm}$ space between the stripes. The rod, mounted on the center of the disk and maintained directly in contact with it, could be independently rotated $360^{\circ}$ around its central axis. Magnets were fixed inside the rod to keep it in contact with the disk and to prevent involuntary deviation from its standard position during haptic scanning. However, a small amount of force was required for its position to be changed intentionally. The disk was graduated in degrees (the sensitivity threshold of this display was equal to $0.25^{\circ}$ ). The rod and the disk were positioned in the frontal plane and were centered on the participant's body midline as he or she remained in a sitting posture during the test. The height of the disk was adjusted with reference to the participant's height, so that the center of the disk was at the level of the participant's solar plexus (see Figure 1).

\section{Procedure}

The participants were taken individually into a quiet room in which the rod device was hidden by a dark cloth; thus, they had no previous visual experience of the test apparatus. After the participants had been blindfolded with a "sleeping" mask (Quies, www. quies.com), the apparatus was uncovered.

Four conditions were studied. It must be noticed that the participants were unaware of the different backgrounds that were pre-

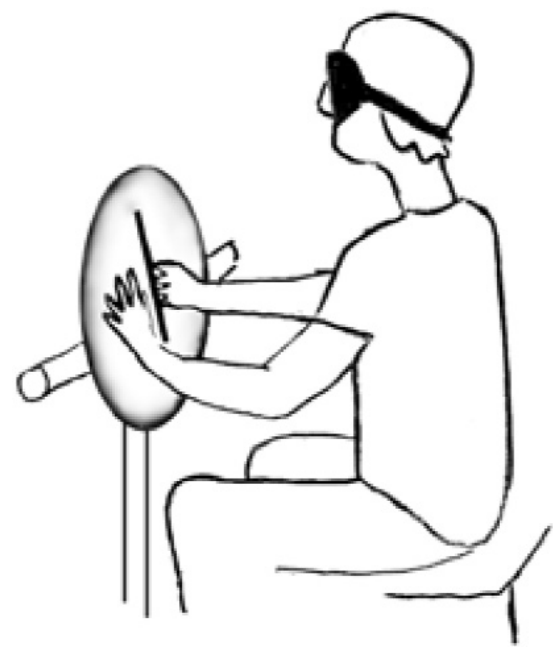

Figure 1. Schema of the test apparatus in the control condition.

sented during the experiment. These conditions, for which the order of presentation were randomized for each subject, were the following.

1. Control condition (no contextual cues). In this condition, a sheet of paper was inserted between the rod and the disk in order to prevent tactile cues for orientation being given to the left hand.

2. Congruent context condition. In this condition, the sheet was taken off, and the stripes covering the metal disk could be perceived by the left hand. The disk was positioned so that the orientation of its stripes were the same as that of the standard $\left(0^{\circ},-45^{\circ}\right.$, or $\left.45^{\circ}\right)$.

3. Left-tilted context condition. In this condition, the stripes covering the metal disk were positioned $22.5^{\circ}$ counterclockwise relative to the standard orientation.

4. Right-tilted context condition. The stripes were clockwise tilted $22.5^{\circ}$ relative to the standard orientation.

In each of these conditions, the participants were instructed to align the rod to three different standard orientations: $0^{\circ}$ (vertical), $-45^{\circ}$, and $+45^{\circ}$. Clockwise tilt from the standard orientation was noted positively, whereas counterclockwise tilt was noted negatively. Gravitational definitions of the vertical were given: "the direction of the rain without wind" and "the direction of a plumbline." Oblique orientations were defined as being the middle of the right angle constituted by the vertical and the horizontal. Six trials were performed for each orientation, and the order of presentation for the 30 orientations was randomized for each participant. During the adjustment, no time limit was imposed, and no feedback was given after the adjustment. The participants were told that the right hand should be used to position the rod without its touching the disk located behind. During the trial, the palm of the left hand was in permanent contact with the disk, exploring with small movements of the fingers the left half of the disk at the left of the rod. A few trials were given in order to familiarize the participants with the instructions, and during the task, the experimenter monitored performance to ensure that the instructions were followed. The experimenter took care that the left hand was always in contact with the disk. The initial rod position was $33.75^{\circ}$ away from the standard orientation, and the direction (counterclockwise and clockwise) was counterbalanced over the six trials.

\section{RESULTS}

Data analyses were carried out on the angular difference (in degrees) between the standard position and the position of the response rod. Thus, the algebraic devia- 
tion from the tested orientations was noted: Deviations to the left (rod turned counterclockwise) were counted as negative, and deviations to the right (clockwise) were counted as positive. Two measures were computed here: the variable error (or within-subjects variability; standard deviation) and the constant error (mean signed errors). As has been noted by Howard (1982), accuracy in judging an orientation is indicated by the mean signed error with which a person is able to set a line to this orientation. A person's precision or sensitivity is indicated by the mean unsigned error or by the standard deviation. Thus, the constant error is the mean of the algebraic differences between the positions of the standard rod and the response rod, calculated over the six trials. This measure can indicate whether a systematic deviation (or bias) of the response is observed and whether this deviation errs in the direction of the contextual stripes in Conditions 3 and 4 . The variable error is the standard deviation computed for each participant over the algebraic deviations obtained during the six trials in each condition. A .05 alpha level was adopted throughout the statistical analyses. The results are summarized in Table 1 .

\section{Variable Errors}

In order to know whether the haptic perception of orientations and, in particular, the classical haptic oblique effect (higher precision in vertical orientation than in $\pm 45^{\circ}$ oblique orientations) was influenced by the nature of the contextual background explored with the left hand, we conducted a 4 (context) $\times 3$ (orientation) analysis of variance (ANOVA), with repeated measures on these factors on the variable error (precision of adjustments).

The main effect of orientations was significant $\left[F(2,38)=62.47, M S_{\mathrm{e}}=3.92\right]$. An oblique effect was evidenced: Post hoc analysis (Newman-Keuls test) showed that the vertical was estimated more precisely $(M=$ $\left.1.892^{\circ}\right)$ than both the $-45^{\circ}$ oblique $\left(M=4.605^{\circ}\right)$ and the $+45^{\circ}$ oblique $\left(M=5.161^{\circ}\right)$ orientation. These two oblique orientations did not differ significantly. Furthermore, the analysis revealed a main effect of context $[F(3,57)=$ $\left.11.97, M S_{\mathrm{e}}=4.374\right]$. The precision in the presence of a congruent context was higher $\left(M=2.754^{\circ}\right)$ than that obtained in the other conditions considered separately (Newman-Keuls test). The precision of the adjustments obtained in the control condition $\left(M=5.025^{\circ}\right)$ was significantly lower, relative to each of the other conditions. A post hoc analysis failed to reveal a significant difference between right- and left-tilted contexts $\left(M=4.023^{\circ}\right.$ and $M=3.742^{\circ}$, respectively).

The interaction between context and orientation factors was also significant $\left[F(6,114)=2.89, M S_{\mathrm{e}}=2.80\right.$; see Figure 2]. The contextual haptic cues did not have the same influence on the perception of the different orientations. The effect was stronger for the oblique orientations than for the vertical. However, preplanned partial analyses showed that, for the vertical orientation, the congruent context significantly improved the precision of adjustment, in contrast to the three other conditions $\left[(\mathrm{F} 1,19)=10.84, M S_{\mathrm{e}}=0.54\right]$. A similar context effect was found for the $-45^{\circ}$ oblique $\left[F(1,19)=14.38, M S_{\mathrm{e}}=\right.$ 4.47] and for the $+45^{\circ}\left[F(1,19)=12.98, M S_{\mathrm{e}}=3.89\right]$ orientations. As a consequence, the oblique effect was weakened in the presence of contextual cues, due to an improvement of oblique precision, particularly in the congruent context condition.

\section{Constant Errors}

In order to investigate whether systematic deviations were present as a function of the tilted background, an analysis was conducted on the constant errors (mean signed errors). A 4 (context) $\times 3$ (orientation) ANOVA, with repeated measures on these factors, revealed a main effect of context condition $\left[F(3,57)=2.81, M S_{\mathrm{e}}=25.38\right]$. Contextual cues led to systematic deviations in the perception of orientations. A post hoc analysis showed that the left-tilted background led to a significant deviation in its direction $\left(M=-1.822^{\circ}\right)$, relative to the right-tilted stripes background $\left(M=0.828^{\circ}\right)$. Neither the main ef-

Table 1

Perception of Orientations (Variable Errors and Constant Errors, in Degrees, With Standard Errors of the Means) as a Function of Orientations and Contexts

\begin{tabular}{|c|c|c|c|c|c|c|c|c|}
\hline \multirow[b]{3}{*}{ Orientation } & \multicolumn{8}{|c|}{ Context } \\
\hline & \multicolumn{2}{|c|}{ Control } & \multicolumn{2}{|c|}{ Congruent } & \multicolumn{2}{|c|}{$\begin{array}{l}\text { Left-Tilted } \\
\text { Context }\end{array}$} & \multicolumn{2}{|c|}{$\begin{array}{l}\text { Right-Tilted } \\
\text { Context }\end{array}$} \\
\hline & $M$ & SEM & $M$ & SEM & $M$ & SEM & $M$ & SEM \\
\hline \multicolumn{9}{|c|}{ Variable Errors } \\
\hline $0^{\mathbf{o}}$ & 2.290 & 0.176 & 1.424 & 0.156 & 1.870 & 0.153 & 1.984 & 0.217 \\
\hline$-45^{\circ}$ & 5.921 & 0.515 & 3.053 & 0.520 & 4.109 & 0.414 & 5.338 & 0.602 \\
\hline$+45^{\circ}$ & 6.864 & 0.522 & 3.784 & 0.621 & 5.248 & 0.557 & 4.747 & 0.534 \\
\hline \multicolumn{9}{|c|}{ Constant Errors } \\
\hline $0^{\circ}$ & -0.150 & 0.418 & -0.367 & 0.541 & -1.900 & 0.765 & -0.229 & 0.625 \\
\hline$-45^{\circ}$ & -1.079 & 1.590 & -0.246 & 1.341 & -2.263 & 2.354 & 1.829 & 1.780 \\
\hline$+45^{\circ}$ & 0.617 & 1.768 & -0.458 & 1.417 & -1.304 & 2.515 & 0.883 & 2.605 \\
\hline
\end{tabular}




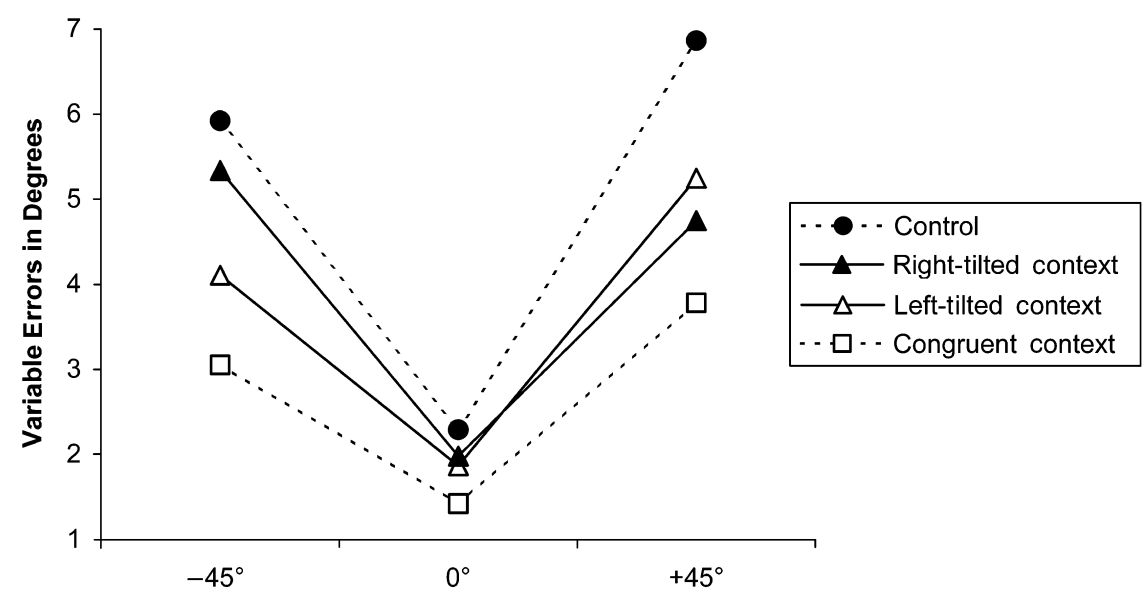

Figure 2. The perception of orientations (variable errors in degrees) as a function of context and orientation factors.

fect of orientation nor the interaction between the two factors was significant $\left[F(2,38)<1, M S_{\mathrm{e}}=157.90\right.$, and $F(6,114)<1, M S_{\mathrm{e}}=34.19$, respectively $]$.

\section{DISCUSSION}

In this research, we have studied the influence of contextual cues on the haptic perception of orientations and the oblique effect. Blindfolded participants had to position a bar with their right hand to three different orientations in the frontoparallel plane: the vertical $\left(0^{\circ}\right)$ and the symmetrical obliques $\left(-45^{\circ}\right.$ and $\left.+45^{\circ}\right)$. During this adjustment, the left hand was touching the background of the half of the disk to the left of the rod. Four contexts were investigated: a smooth background (control condition), a congruent context (the stripes engraved on the disk were parallel to the orientation to be estimated), and two incongruent contexts in which the stripes were tilted to the left and to the right of the participant, respectively, relative to the standard orientation.

Concerning the precision of adjustments (variable errors), the oblique effect obtained in the control condition (no context) was similar to that obtained previously by Gentaz et al. (2002) with a similar method of direct estimation of orientations (production task). Indeed, most experiments on the haptic oblique effect have been carried out with a reproduction task in which an unknown but previously scanned orientation had to be memorized. The present experiment confirms that a haptic oblique effect in the frontoparallel plane can be evidenced with a production task that involves a cognitive representation of orientations, probably on the basis of an internal model of gravity. More interesting is the fact that tactile contextual cues had an influence on the production of oblique and vertical orientations, particularly on the oblique effect. When the context was congruent with the standard orientation, the precision of adjustments (variable error) was improved, an effect very similar to the effect exerted by visual vertical cues on spatial orientation (see the introduction). During incongruent context scanning, the precision of orientations, particularly for the obliques, also tended to be enhanced, and as a consequence, the oblique effect was significantly weakened. However, this improvement does not mean that accuracy was enhanced, since an analysis of constant error revealed that deviations erred in the direction of the stripes. Thus, the presence of a context, congruent or not, diminishes the variability of estimations and, therefore, enhances the precision of the production of orientations. Furthermore, the mismatch between the orientation to be produced and the context (incongruent condition) revealed a tendency to adjust the rod in the direction of this context. These overall results fit well with a haptic frame of reference that can be used in determining orientations in space, although further experiments are needed to rule out a congruency effect, a direct influence of the context on the perceived rod. Further experiments are needed to address these hypotheses more clearly - in particular, by studying the complete function of the inclination of a context and by using a more structured pattern (oriented frame).

Another interesting aspect of these results is the fact that the contextual cues picked up by the left hand were processed, since the haptic estimation of orientations made by the right hand was affected. As was noted previously, the haptic sense picks up information in external space in a sequential way, at least when only one hand is used. This sequential exploration can weaken or eliminate an effect of the context. However, this research shows that when both hands are used simultaneously, the left one exploring the background and the other orienting the stimulus rod, information provided by the textured background is picked up by the left hand. This information is then processed at high levels of the tactile information system and integrated into the sensorimotor 
process controlling the right hand. As a consequence, contextual cues influence the perception of the oriented stimulus rod. Thus, when hands simultaneously explore external space, context effects similar to those found in vision, even though different central processes are relied on, are likely to appear.

\section{REFERENCES}

Appelle, S. (1972). Perception and discrimination as a function of stimulus orientation: The "oblique effect" in man and animals. Psychological Bulletin, 78, 266-278.

ApPelle, S., \& Countryman, M. (1986). Eliminating the haptic oblique effect: Influence of scanning incongruity and prior knowledge of the standards. Perception, 15, 325-329.

Attneave, F., \& Olson, R. K. (1967). Discriminability of stimuli varying in physical and retinal orientation. Journal of Experimental Psychology, 74, 149-157.

Attneave, F., \& ReID, K. W. (1968). Voluntary control of frame of reference and slope equivalence under head rotation. Journal of Experimental Psychology, 78, 153-159.

BANKS, M. S., \& STOLARZ, S. J. (1975). The effect of head tilt on meridional differences in acuity: Implications for orientation constancy. Perception \& Psychophysics, 17, 17-22.

Beh, H. C., Wenderoth, P. M., \& Purcell, A. T. (1971). The angular function of a rod-and-frame illusion. Perception \& Psychophysics, 9(4), 353-355.

CHEN, S., \& LEVI, D. M. (1996). Meridional anisotropy in the discrimination of parallel and perpendicular lines: Effect of body tilt. Perception, 25, 633-649.

Corwin, T. R., Moskowitz-Cook, A., \& Green, M. A. (1977). The oblique effect in a vernier acuity situation. Perception \& Psychophysics, 21, 445-449.

Essock, E. A. (1980). The oblique effect of stimulus identification considered with respect to two classes of oblique effects. Perception, 9 , 37-46.

Essock, E. A., Krebs, W. K., \& Prather, J. R. (1997). Superior sensitivity for tactile stimuli oriented proximally-distally on the finger: Implications for mixed class 1 and class 2 anisotropies. Journal of Experimental Psychology: Human Perception \& Performance, 23, 515-527.

Foster, D., \& Westland, S. (1998). Multiple groups of orientationselective visual mechanisms underlying rapid orientation-line detection. Proceedings of the Royal Society of London: Series B, 256, 16051613.

Furmanski, C. S., \& ENGEL, S. A. (2000). An oblique effect in human primary visual cortex. Nature Neuroscience, 3, 535-536.

Gentaz, E. (2000). Existe-t-il un "effet de l'oblique" dans la perception tactile des orientations? [Is there an oblique effect in the tactual perception of orientations?]. L'Année Psychologique, 100, 111-140.

Gentaz, E., Badan, M., Luyat, M., \& Touil, N. (2002). The manual haptic perception of orientations and the oblique effect in patients with left visuo-spatial neglect. NeuroReport, 13, 327-331.

Gentaz, E., \& Ballaz, C. (2000). La perception visuelle des orientations et l'effet de l'oblique [The visual perception of orientations and the oblique effect]. L'Année Psychologique, 100, 715-744.

Gentaz, E., \& Hatwell, Y. (1995). The haptic "oblique effect" in children's and adults' perception of orientation. Perception, 24, 631-646.

Gentaz, E., \& Hatwell, Y. (1996). Role of gravitational cues in the haptic perception of orientation. Perception \& Psychophysics, 58, 1278-1292.

GentaZ, E., \& Hatwell, Y. (1998). The haptic oblique effect in the perception of rod orientation by blind adults. Perception \& Psychophysics, 60, 157-167.

GENTAZ, E., \& HATWELL, Y. (1999). Role of memorisation conditions in the haptic processing of orientations and the "oblique effect." British Journal of Psychology, 90, 373-388.
Gentaz, E., \& Hatwell, Y. (2004). Geometrical haptic illusions: The role of exploration in the Müller-Lyer, vertical-horizontal, and Delboeuf illusions. Psychonomic Bulletin \& Review, 11, 31-40.

Gentaz, E., Luyat, M., Cian, C., Hatwell, Y., Barraud, P.-A., \& RAPHEL, C. (2001). The reproduction of vertical and oblique orientations in the visual, haptic, and somato-vestibular systems. Quarterly Journal of Experimental Psychology, 54A, 513-526.

Gentaz, E., \& Streri, A. (2004). An “oblique effect" in infants' haptic perception of spatial orientations. Journal of Cognitive Neuroscience, 16, 253-259.

GENTAZ, E., \& TsCHOPP, C. (2002). The oblique effect in the visual perception of orientations. In P. Shovoh (Ed.), Advances in psychology research (pp. 137-163). New York: Nova Sciences.

Groen, E. L., Jenkin, H. L., \& Howard, I. P. (2002). Perception of selftilt in a true and illusory vertical plane. Perception, 31, 1477-1490.

Heeley, D. W., \& Buchanan-Smith, H. M. (1990). Recognition of stimulus orientation. Vision Research, 30, 1429-1437.

Hermens, F., \& Gielen, S. (2003). Visual and haptic matching of perceived orientations of lines. Perception, 32, 235-248.

HowARD, I. P. (1982). Human visual orientation. New York: Wiley.

HowARD, I. P., \& Hu, G. (2001). Visually induced reorientation illusions. Perception, 30, 583-600.

Kappers, A. M. (1999). Large systematic deviations in the haptic perception of parallelity. Perception, 28, 1001-1012.

Kappers, A. M., \& KoENDERINK, J. J. (1999). Haptic perception of spatial relations. Perception, 28, 781-795.

Lechelt, E. C., \& VerEnKA, A. (1980). Spatial anisotropy in intramodal and cross-modal judgments of stimulus orientation: The stability of the oblique effect. Perception, 9, 581-589.

LENNIE, P. (1974). Head orientation and meridional variations in acuity. Vision Research, 14, 107-111.

Li, B., Peterson, M. R., \& Freeman, R. D. (2003). Oblique effect: A neural basis in the visual cortex. Journal of Neurophysiology, 90, 204-217.

LUYAT, M., \& GENTAZ, E. (2002). Body tilt effect on the reproduction of orientation: Studies on the visual oblique effect and subjective orientations. Journal of Experimental Psychology: Human Perception \& Performance, 28, 1002-1011.

LuYat, M., Gentaz, E., Regia Corte, T., \& Guerraz, M. (2001). Reference frames and haptic perception of orientation: Body and head tilt effects on the oblique effect. Perception \& Psychophysics, 63, 541-554.

Luyat, M., \& OHLMann, T. (1997). Effet d'un contexte visuel incliné: Vers une explication en termes d'inclinaison du plan médian apparent [The effect of a visual tilted context: Toward a straight-ahead shift hypothesis]. L'Année Psychologique, 97, 267-292.

Luyat, M., Ohlmann, T., \& Barraud, P.-A. (1997). Subjective vertical and postural activity. Acta Psychologica, 95, 181-193.

McIntyre, J., Lipshits, M., ZAOUI, M., Berthoz, A., \& GURFinkel, V. (2001). Internal reference frames for representation and storage of visual information: The role of gravity. Acta Astronautica, 49, 111-121.

McMahon, M. J., \& MacLeod, D. I. (2003). The origin of the oblique effect examined with pattern adaptation and masking. Journal of Vision, 3, 230-239.

Morgan, M. J. (1991). Hyperacuity. In D. M. Regan (Ed.), Spatial vision (pp. 87-113). London: Macmillan.

OlDFIELD, R. C. (1971). The assessment and analysis of handedness: The Edinburgh inventory. Neuropsychologia, 9, 97-113.

Orban, G. A., Vandenbussche, E., \& Vogels, R. (1984). Human orientation discrimination tested with long stimuli. Vision Research, 24, 121-128.

ReGAN, D., \& Price, P. (1986). Periodicity in orientation discrimination and the unconfounding of visual orientation. Vision Research, 26, 1299-1302.

RocK, I. (1990). The frame of reference. In I. Rock (Ed.), The legacy of Solomon Asch (pp. 243-268). Hillsdale, NJ: Erlbaum.

SAARINEN, J., \& LEVI, D. (1995). Orientation anisotropy in vernier acuity. Vision Research, 35, 2449-2461.

Spinelli, D., Antonucci, G., Goodenough, D. R., Pizzamiglio, L., \& ZoccolotTi, P. (1991). Psychophysiological mechanisms under- 
lying the rod and frame illusion. In S. Wapner \& J. Demick (Eds.), Field dependence-independence: Cognitive style across the life span (pp. 37-60). Hillsdale, NJ: Erlbaum.

WADE, N. J. (1992). The representation of orientation in vision. Australian Journal of Psychology, 44, 139-145.
WaLker, J. T. (1972). Tactual field dependence. Psychonomic Science, 26, 311-313.

Westheimer, G. (2003). Meridional anisotropy in visual processing: Implications for a neural site of the oblique effect. Vision Research, $\underline{43,2281-2289 .}$.

(Manuscript received December 12, 2003;

revision accepted for publication October 13, 2004.) 